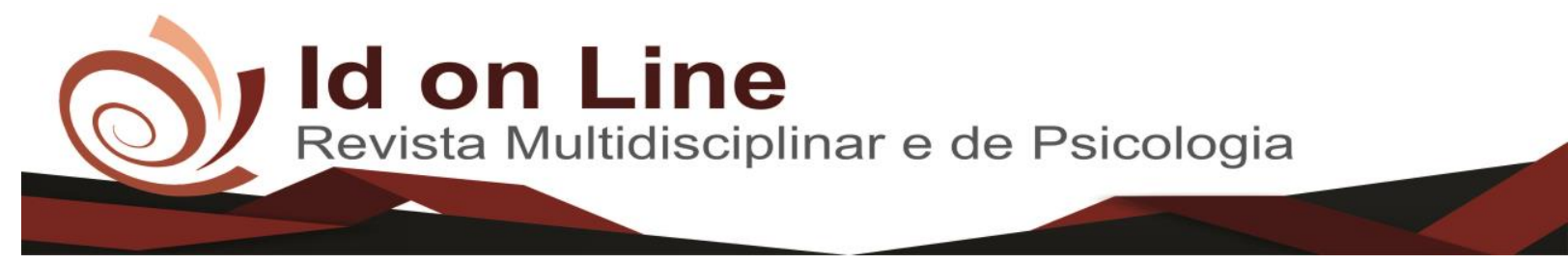

Artigo de Revisão

\title{
Peeling de Ácido Salicílico no Tratamento da Acne: Revisão Baseada em Evidências Clínicas
}

\author{
Bruna Luana Sousa Cunha ${ }^{1}$, Lilian Abreu Ferreira ${ }^{2}$
}

\begin{abstract}
Resumo: A acne é uma doença genética hormonal que pode, se não adequadamente tratada, causar alterações inestéticas na pele. Entre as opções de tratamento, o peeling de ácido salicílico é um procedimento amplamente utilizado, capaz de esfoliar e remover superficialmente lesões, promovendo a regeneração de novos tecidos cutâneos. Trata-se de uma revisão integrativa sobre a ação do peeling de ácido salicílico no tratamento da acne, realizada através de busca na base Pubmed nos últimos 10 anos. O peeling de ácido salicílico mostrou-se eficaz tanto em lesões não inflamatórias quanto inflamatórias. Quando comparado a outros peelings, como o de ácido tricloracético, ácido pirúvico e a procedimentos como a fototerapia, mostrou resultados semelhantes. Mostrou ainda, resultados melhores quando comparado ao peeling de Jessner e de ácido mandélico. Assim, como a acne traz insatisfações ao indivíduo acometido, o peeling de ácido salicílico é uma opção de tratamento efetivo e seguro, como evidenciado nos estudos encontrados.
\end{abstract}

Palavras-chave: Abrasão Química; Ácido Salicílico; Acne Vulgar.

\section{Salicylic Acid Peeling in Acne Treatment: Review Based on Clinical Evidence}

\begin{abstract}
Acne is a genetic hormonal disease that can cause unsightly changes in the skin, if not properly treated. Among the treatment options, salicylic acid peeling is a widely used procedure, capable of exfoliating and superficially removing lesions and promoting the regeneration of new cutaneous tissues. This is an integrative review on the action of salicylic acid peeling in the treatment of acne, conducted through Pubmed search in the last 10 years. Salicylic acid peeling has been shown to be effective in both non-inflammatory and inflammatory lesions. When compared to other peelings such as trichloracetic acid, pyruvic acid and procedures such as phototherapy, it showed similar results. It also showed better results when compared to Jessner's peeling and mandelic acid. Thus, as acne brings dissatisfaction to the affected individual, salicylic acid peeling is an effective and safe treatment option, as evidenced in the studies found.
\end{abstract}

Keywords: Chemexfoliation; Salicylic Acid; Acne Vulgaris.

\footnotetext{
${ }^{1}$ Curso de Farmácia. Faculdade Patos de Minas. Contato: vtolentino@ hotmail.com;

${ }^{2}$ Graduada em Farmácia pelo Centro Universitário de Patos de Minas. Mestre em Ciências da Saúde com área de concentração em Farmoepidemiologia pelo Instituto de Previdência dos Servidores de Minas Gerais (2010). Especialista em Farmacologia Clínica pela Universidade Estadual de Montes Claros (2008). Especialista em Manipulação Magistral Alopática pelo Centro Universitário de Patos de Minas (2007). Atualmente é Professora do departamento de Farmácia da Faculdade de Patos de Minas. Contato: lyabreu@ hotmail.com
} 


\section{Introdução}

A aparência é um aspecto importante e influencia a maneira pela qual somos percebidos pelos outros. Como parte de nossa rotina, os itens de perfumaria e cosméticos são considerados essenciais para a saúde, autoestima e bem-estar. Como a pele é o órgão mais visível do corpo, as dermatoses na região facial podem ter impacto negativo, tanto na aceitação de sua própria imagem quanto na qualidade de vida (ABIHPEC, 2016; VILAR; SANTOS; SOBRAL FILHO, 2015; SELORES, 2011).

Assim, disfunções estéticas, como por exemplo a acne, podem acarretar grandes insatisfações pessoais. A acne vulgar é uma desordem crônica pilossebácea causada por descamação anormal do epitélio folicular. Isso leva à obstrução do canal pilossebáceo, resultando em inflamação e subsequentemente a formação de pápulas, pústulas, nódulos, comedões e cicatrizes. A atividade excessiva das glândulas sebáceas e o bloqueio nos dutos leva à formação de comedões que mais tarde inflamam, pelo crescimento anormal da bactéria Propionibacterium acnes (AL-TALIB et al., 2017).

Embora não seja comumente considerada uma afecção grave, pode ser muito incômoda e, dependendo do grau, causa alterações estéticas que podem ter impacto social e psicológico na qualidade de vida do indivíduo. Ela acomete cerca de $80 \%$ dos adolescentes e persiste até a idade adulta entre $40 \%$ dos indivíduos, sendo o rosto a parte mais atingida (ARAUJO; BRITO, 2017; KRUNGER; KUMAR, 2012; SELORES, 2011). O impacto na qualidade de vida que a acne pode apresentar justifica o seu tratamento adequado, para evitar consequências emocionais e persistentes complicações (GUPTA et al., 2016).

Entre as opções de tratamento estão a limpeza de pele, cosméticos, medicamentos antissépticos e anti-inflamatórios, e a utilização de procedimentos estéticos, como os peelings, para melhorar a aparência (ARAÚJO; BRITO, 2017). O peeling químico consiste na aplicação de um agente químico na pele que promove a destruição de uma toda ou parte da epiderme, com ou sem aprofundamento na derme. É um procedimento amplamente utilizado no manejo da acne, pois é capaz de esfoliar e remover superficialmente lesões, e promover a regeneração de novos tecidos cutâneos (KONTOCHRISTOPOULOS; PLATSIDAKI, 2017; FASIH; ARIF, 2016). 
A profundidade do peeling vai depender do tipo de pele, tratamentos prévios, local, técnica de aplicação e agente utilizado, entre outros, sendo classificados em peeling muito superficial (camada granulosa e córnea), superficial (epiderme), médio (derme papilar) e profundo (derme reticular) (BAGATIN; HASSUN; TALARICO, 2009).

Entre os agentes químicos mais frequentemente usados, está o ácido salicílico (KONTOCHRISTOPOULOS; PLATSIDAKI, 2017; FASIH; ARIF, 2016), um beta hidroxiácido, amplamente utilizado em peelings superficiais devido ao seu poder esfoliativo, queratolítico e queratoplástico. O peeling de ácido salicílico é a escolha para o tratamento da acne ativa, devido ao seu alto poder comedolítico e efeito sebostático, promovendo descamação da parte superior das camadas lipídicas do estrato córneo por meio da sua capacidade de dissolver o cimento intercelular, reduzindo assim a adesão dos corneócitos. Devido à sua lipofilicidade, apresenta uma melhor penetração na unidade pilossebácea; além disso, as camadas mais externas da pele possuem grandes concentrações lipídicas, o que permite que o ácido salicílico aja e promova a remoção das células mortas (BAUMANN; SAGHARI, 2009).

Assim, o objetivo do estudo foi realizar uma revisão integrativa sobre a ação do peeling de ácido salicílico no tratamento da acne.

\section{Método}

Foi realizada uma revisão integrativa através de uma busca de artigos na base PubMed, utilizando as palavras-chave "acne", "chemical peeling" e "salicylic acid". Foram incluídos ensaios clínicos que utilizaram o peeling de ácido salicílico no tratamento facial da acne. Foram selecionados artigos publicados no período de 2007 a 2018, nas línguas Portuguesa, Inglesa e Espanhola. Não foram incluídos estudos in vitro, realizados em animais e estudos com associação de ativos juntamente com o ácido salicílico ou uso de qualquer outra terapia ou procedimento coadjuvante ao ácido salicílico.

Inicialmente, para melhor compreensão do tema abordado foi apresentado um breve resumo sobre as características da acne, as definições sobre peelings químicos e as principais características e ação do ácido salicílico sobre a pele. 
A acne vulgar é uma afecção inflamatória crônica dos folículos pilossebáceos causada por uma descamação anormal do epitélio folicular e atividade excessiva da glândula sebácea, o que leva à obstrução do canal folicular resultando na inflamação e formação de pápulas, pústulas, nódulos e cistos que podem levar a formação de cicatrizes permanentes. É uma condição extremamente comum que acomete $85 \%$ dos adolescentes e adultos jovens atingindo principalmente o rosto, a parte superior das costas e do peito. É mais predominante no sexo masculino, porém é mais persistente no feminino (AL-TALIB et al., 2017; FASIH; ARIF, 2016).

Vários outros fatores também interferem na predisposição da acne, como os aspectos genéticos, hormonais, uso de medicamentos como anabolizantes, anticoncepcionais orais, corticosteroides tópicos e orais, lítio e isoniazida e aumento da colonização da bactéria Propionibacterium acnes (P. acnes) no canal. A hiperestimulação androgênica leva a um aumento de sebo que fica retido, propiciando a colonização pela bactéria (ARAUJO; BRITO, 2017).

Conforme o tipo de lesão, a acne pode ser classificada em Grau I, não inflamatória, apresentando apenas comedões fechados e abertos; Grau II, - presença de lesões inflamatórias (pápulas/pústulas) com numerosos comedões; Grau III, presença de nódulos e cistos, além de comedões, pápulas e pústulas; e o Grau IV que é uma forma mais severa, com múltiplas lesões inflamatórias, abscessos e fístulas com evolução para lesões cicatriciais (KATARIA; CHHILLAR, 2015).

A acne vulgar pode influenciar muito a qualidade de vida de uma pessoa, portanto, é necessário um tratamento adequado para evitar consequências emocionais e persistentes complicações (AL-TALIB et al., 2017; GUPTA et al., 2016; SELORES, 2011).

Muitos profissionais de saúde sugerem o uso de peelings químicos superficiais como uma opção de tratamento para a acne vulgar, pois é um procedimento relativamente seguro. Além disso, várias outras opções de tratamento estão disponíveis, mas exigem um longo tempo para produzir resultados efetivos (FASIH; ARIF, 2016). Muito embora existam muitos agentes tópicos e sistêmicos utilizados no tratamento da acne, a adesão a essas terapias é baixa devido 
ao tempo de tratamento prolongado e aos seus efeitos colaterais (SHARQUIE; NOAIMI; ALJANABI, 2014).

É necessária uma terapia adicional eficaz que acelere a recuperação, como o uso de peelings superficiais (AL-TALIB et al., 2017; FASIH; ARIF, 2016). Os peelings químicos superficiais permanecem como padrão para o tratamento de cicatrizes da acne, linhas finas de expressão, hiperpigmentação, entre outros (AL-TALIB et al., 2017).

\section{Peelings Químicos}

A técnica consiste na aplicação de um agente químico que provoca uma destruição controlada de parte da epiderme ou de toda ela, com ou sem penetração na derme, levando à esfoliação e remoção de lesões superficiais, seguida de regeneração de todo o tecido (FASIH; ARIF, 2016).

Os peelings podem ser classificados quanto ao nível de profundidade em peelings muito superficiais ou superficiais, que atingem somente a epiderme - neste tipo de peeling, o melhor resultado é obtido com aplicações em séries com intervalos curtos. A descamação é frequentemente fina e clara, não alterando a rotina diária do paciente. Este tipo de peeling melhora a textura da pele, são coadjuvantes no tratamento da acne ativa, clareia manchas e atenua rugas finas, além de estimular a renovação do colágeno. Em peelings médios, que provocam descamação mais profunda e escura, o que pode exigir do paciente sete a 15 dias para retorno à rotina diária. Estes são indicados para ceratoses (lesões pré-cancerosas) e rugas mais pronunciadas. E por fim, em peelings profundos, que são mais fortes e agressivos que os demais. Provocam a formação de muitas crostas espessas, e o pós peeling pode exigir o uso de curativos. A recuperação pode durar até três meses. Apresentam resultados significativos, com renovação importante da pele e diminuição de rugas profundas, como aquelas ao redor da boca e dos olhos (KONTOCHRISTOPOULOS; PLATSIDAKI, 2017; YOKOMIZO et al, 2013).

O peeling pode ser utilizado na prática clínica para reduzir também manchas e cicatrizes mais superficiais da acne. No tratamento da acne ativa, o peeling químico superficial irá colapsar os corneócitos, esfoliar camadas superficiais de epiderme e aumentar a atividade epidérmica das enzimas, além de diminuir a porcentagem das lesões (AL-TALIB et al., 2017). 
É um procedimento utilizado por profissionais de saúde da área estética como forma direta de limpeza, a fim de retirar as impurezas presentes, como os comedões, para impedir a oclusão dos folículos pilossebáceos e retirar a oleosidade da pele, principalmente em adolescentes que apresentam maiores níveis de hormônios androgênicos, o que leva ao maior acúmulo de sebo devido à hiperatividade das glândulas sebáceas (ARAUJO; BRITO, 2017).

O ácido salicílico é amplamente utilizado em peelings superficiais devido ao seu poder esfoliativo, queratolítico e queratoplástico (KONTOCHRISTOPOULOS; PLATSIDAKI, 2017; FASIH; ARIF, 2016).

\section{Peeling de Ácido Salicílico}

O ácido salicílico (AS) é um membro de um grupo de compostos conhecidos como hidroxiácidos, que são amplamente utilizados para uma série de indicações cosméticas devido às suas muitas propriedades importantes. É usado topicamente para tratar várias afecções da pele por mais de 2000 anos. Por sua ação comedolítica, antiinflamatória e queratolítica, o AS é utilizado em muitas formulações tópicas para tratamento da acne. Ele também é capaz de aumentar a penetração de outros agentes tópicos. Quimicamente, é um beta-hidroxiácido ou ácido 2-hidroxibenzóico que possui um anel fenólico em sua estrutura. As principais fontes naturais de AS e salicilatos incluem folhas e cascas de salgueiro, bétula e gaultéria. No entanto, o AS também pode ser sintetizado artificialmente (ARIF, 2015).

O AS é um agente lipossolúvel, sendo, portanto, miscível aos lipídeos epidérmicos e das glândulas sebáceas nos folículos pilosos, apresentando melhor penetração (FASIH; ARIF, 2016; AL-TALIB et al., 2017).

O AS diminui a adesão de corneócitos, pois possui capacidade de dissolver o cimento intercelular. A atividade antiinflamatória do ácido salicílico torna-o útil na redução rápida do eritema facial. O ácido salicílico também tem um perfil de segurança muito bom, sendo que a sua toxicidade (salicilismo) ocorre quando aplicado a grandes áreas devido à absorção sistêmica, e é geralmente associada a altas concentrações. É de baixo custo, fácil de aplicar e tem a capacidade de auto-neutralização. Outro benefício é o seu efeito clareador na pigmentação pós-inflamatória devido à acne (KONTOCHRISTOPOULOS; PLATSIDAKI, 2017; ALTALIB et al., 2017). 
Antes da aplicação do peeling, a avaliação da pele do indivíduo é obrigatória antes de considerar o peeling químico. O profissional esteta deve avaliar o paciente quanto às possíveis indicações, contraindicações, riscos e limitações da técnica, discutir detalhadamente o procedimento e avaliar as expectativas do paciente. Além disso, é importante realizar um teste de sensibilidade ao ácido com uma formulação a 20\% (ARIF, 2015).

Em pacientes com acne vulgar, tratamentos tópicos e sistêmicos podem ser iniciados entre 2 a 4 semanas antes do peeling. O peróxido de benzoíla tópico e outras formulações antibióticas tópicas podem ser usadas diariamente e descontinuadas um ou dois dias antes do procedimento. A profilaxia antiviral é recomendada em peelings médios e profundos, entretanto, como o peeling de ácido salicílico é superficial, deve-se avaliar a necessidade da terapia pelo histórico de doenças do paciente. Dois dias antes ou no dia do peeling químico, inicia-se a profilaxia antiviral que é continuada por 7 a 10 dias (ARIF, 2015; TOSTI; GRIMES; PADOVA, 2006). A concentração padrão para o peeling químico com o ácido salicilico é de $20 \%$ ou $30 \%$ em uma base alcoólica. A tabela 1 mostra as várias concentrações do ácido salicílico e suas respectivas indicações (ARIF, 2015).

Tabela 1 - Concentrações do ácido salicílico e suas respectivas indicações

\begin{tabular}{c|c}
\hline Concentração & Indicação \\
\hline $0,5 \%$ a $10 \%$ & Acne \\
$3 \%-6 \%$ & $\begin{array}{c}\text { Distúrbios hiperqueratóticos, como } \\
\text { psoríase, ictiose, queratose pilar } \\
5 \% \text { a } 40 \%\end{array}$ \\
$50 \%$ & Verrugas, calos \\
$20 \%$ a $30 \%$ & Lesões actínicas e lesões pigmentadas \\
\hline
\end{tabular}

Fonte: ARIF, 2015

O peeling é repetido em um intervalo de 2 a 4 semanas. Os resultados de valor máximo são visíveis após 3 a 6 sessões, dependendo da gravidade, condição e tipo de pele a ser tratada. O peeling ficará em contato com a pele por 3 a 5 minutos, para então ser removido com água abundante. Um sabonete suave pode ser usado para remover qualquer precipitado residual de ácido salicílico. Depois de enxaguar, um hidratante suave é aplicado à pele (ARIF, 2015; KHUNGER; KUMAR, 2012; TOSTI; GRIMES; PADOVA, 2006). 
Após 48 horas o paciente pode retomar suas formulações tópicas de cuidados com a pele, incluindo os retinóides e os agentes antiacneicos. A descamação excessiva e irritação no período pós-peeling pode ser tratada com esteróides tópicos, pois atenuam a inflamação e reduzem a hiperpigmentação pós-inflamatória (ARIF, 2015).

As complicações que podem ocorrer com o peeling de ácido salicílico são leves e transitórias e incluem eritema, secura e sensação de queimação. Seu uso não é recomendado durante a gravidez e é contraindicado em casos de alergia (aspirina) devido ao risco de salicilismo (KONTOCHRISTOPOULOS; PLATSIDAKI, 2017; ARIF, 2015).

\section{Resultados e Discussão}

De acordo com os critérios de inclusão/exclusão, foram incluídos sete estudos na revisão. As principais características e resultados coletados desses estudos foram sumarizadas no Quadro 1.

Dayal e colaboradores compararam a eficácia e a segurança do peeling de ácido salicílico a $30 \%$ versus peeling de Jessner no tratamento da acne facial leve a moderada em pacientes indianos por meio de um ensaio clínico, prospectivo e randomizado. Foram incluídos 40 pacientes com acne vulgar de grau I ao II divididos aleatoriamente em dois grupos. No grupo 1, foi utilizado peeling de ácido salicílico a 30\% e no grupo 2, foi utilizada a solução de Jessner com 2 semanas de intervalo, e um total de seis peelings em cada grupo. Ao final de 12 semanas, a melhora foi significativamente maior no grupo 1. Em relação aos comedões, houve diminuição significativa na contagem média do grupo $1(\mathrm{p}<0,05)$, enquanto que no grupo 2 , não houve diminuição significativa $(\mathrm{p}=0,06)$. Essa diminuição significativa no grupo 1 foi alcançada em 6 semanas, o que não foi alcançada no grupo 2, mesmo ao final de 12 semanas. Já em relação às pápulas, houve diminuição significativa da contagem média em ambos os grupos, mas essa diminuição foi observada mais cedo, às quatro semanas no grupo 1. Em relação às pústulas, houve diminuição significativa da contagem média em ambos os grupos e essa diminuição foi observada às 4 semanas em ambos os grupos. A porcentagem de diminuição no grupo 1 (70,28\%) não foi estatisticamente diferente em relação ao grupo 2 (76,70\%), (p = 0,57). Ambos os peelings foram bem tolerados pelos pacientes. A sensação de queimação e 
ardência no pós-peeling foram os efeitos colaterais mais comuns. Apenas um paciente no grupo 1 e três do grupo 2 apresentaram hiperpigmentação pós-inflamatória. Os autores concluíram que o peeling de ácido salicílico a 30\% é mais eficaz que o peeling de Jessner no tratamento de lesões não inflamatórias (comedões) e na melhora de um modo geral da acne facial leve a moderada (DAYAL et al., 2016).

Também com o objetivo de comparar a eficácia e segurança do peeling de ácido salicílico com a solução de Jessner, um ensaio clínico randomizado e simples-cego foi conduzido em treze pacientes com acne facial. O peeling de Jessner foi aplicado a um lado da face de cada paciente e o peeling de ácido salicílico a 30\% ao outro, em três sessões com intervalos de duas semanas. Um dermatologista cego avaliou e contou lesões não inflamatórias e inflamatórias antes do tratamento e duas semanas após cada tratamento. A contagem de lesões inflamatórias da acne não diferiu significativamente entre o ácido salicílico e a solução de Jessner, embora em termos de contagem de lesões não-inflamatórias, o lado tratado com ácido salicílico mostrou melhora significativa $(\mathrm{p}=0,04)$, enquanto que o lado com a solução de Jessner não. Nenhuma hiperpigmentação pós-inflamatória, cicatriz ou eritema prolongado foi notado após qualquer tratamento (BAE et al., 2013).

Alba e colaboradores realizaram um estudo longitudinal que teve como objetivo avaliar o progresso do tratamento da acne vulgar comparando o peeling de ácido salicílico a $10 \%$ (grupo I) (creme-gel) e a fototerapia (LED azul $470 \mathrm{~nm}$ e laser vermelho $660 \mathrm{~nm}$ ) (grupo II). Participaram do estudo 22 adolescentes com idade entre 12 a 18 anos com acne grau I e II. Ambos os protocolos foram feitos semanalmente em um total de dez sessões. O número de comedões, pápulas e pústulas foram contados no início e no final do estudo, e o resultado determinado pela análise comparativa das fotografias por dois examinadores cegos. Ambas as terapias foram eficazes para o tratamento da acne $(\mathrm{p}<0,01)$. No entanto, quando os dois tratamentos foram comparados, a fototerapia mostrou uma diferença significativa maior na redução do número de pústulas $(\mathrm{p}<0,05)$. O uso da fototerapia, no entanto, devido às suas propriedades antiinflamatórias e cicatrizantes foi mais efetivo na redução do número de pústulas (ALBA et al., 2016).

A eficácia do peeling de ácido pirúvico e do ácido salicílico foi comparada no tratamento da acne leve a moderada em um ensaio clínico prospectivo, simples-cego. Foram incluídos 86 pacientes com acne divididos aleatoriamente em dois grupos (grupo 1 - ácido salicílico a 30\%; grupo 2 - ácido pirúvico a 50\%). Os peelings foram aplicados com um 
intervalo de duas semanas em um total de oito sessões e a avaliação da melhora foi realizada por meio de um algoritmo - índice de gravidade da acne calculado antes do tratamento e nas semanas 2, 4, 6 e 8. Em ambos os grupos, a redução no número de comedões, pápulas e o índice de gravidade da acne foi estatisticamente significativa $(\mathrm{p}<0,001)$. No entanto, não foi significativa em relação ao número de pústulas $(\mathrm{p}=0,09)$. As complicações, queimação, vermelhidão e descamação foram as mais comuns e equivalentes entre os grupos, porém na quinta sessão, foi significativamente menor no grupo $1(\mathrm{p}=0,015)$. Em cada sessão, a queimação ocorreu em $85-100 \%$ dos pacientes, que foi resolvida imediatamente usando bicarbonato de sódio a $10 \%$. Os autores concluíram que os dois peelings são eficazes na melhora da acne leve a moderada, sem diferença significativa na eficácia e nos efeitos colaterais (JAFFARY et al., 2016). 


\begin{tabular}{|c|c|c|c|c|c|}
\hline Autor & $\begin{array}{c}\text { Delineamento } \\
\text { do } \\
\text { estudo }\end{array}$ & $\begin{array}{c}\mathbf{N}^{\mathbf{o}} \text { de } \\
\text { indivíduos }\end{array}$ & Protocolo & $\begin{array}{c}\text { Sessões } \\
\text { (intervalo) }\end{array}$ & Resultado \\
\hline $\begin{array}{l}\text { Abdel } \\
2015\end{array}$ & \begin{tabular}{|l|} 
Ensaio clínico \\
prospectivo, \\
unicêntrico, \\
randomizado \\
e duplo cego
\end{tabular} & 20 & $\begin{array}{l}\text { Peeling de ácido } \\
\text { tricloroacético (ATA) } \\
\text { a 25\% versus peeling } \\
\text { de ácido salicílico a } \\
30 \%\end{array}$ & $\begin{array}{c}5 \\
(2 \text { semanas) }\end{array}$ & $\begin{array}{l}\text { O ácido tricloroacético foi } \\
\text { superior no tratamento das } \\
\text { lesões comedonais, enquanto } \\
\text { o salicílico foi superior no } \\
\text { tratamento de lesões } \\
\text { inflamatórias. Não houve } \\
\text { diferença significativa entre } \\
\text { os dois tratamentos, sendo os } \\
\text { resultados semelhantes. }\end{array}$ \\
\hline $\begin{array}{l}\text { Alba et } \\
\text { al., } 2016\end{array}$ & $\begin{array}{l}\text { Estudo } \\
\text { longitudinal }\end{array}$ & 22 & $\begin{array}{l}\text { Peeling de ácido } \\
\text { salicílico a } 10 \% \text { e } \\
\text { fototerapia com LED- } \\
\text { Laser }\end{array}$ & $\begin{array}{c}10 \\
\text { (semanal) }\end{array}$ & $\begin{array}{l}\text { Ambas as terapias foram } \\
\text { eficazes para o tratamento da } \\
\text { acne }(\mathrm{p}<0,01) \text {. A fototerapia } \\
\text { foi mais efetiva na redução do } \\
\text { número de pústulas ( }<< \\
0,05) \text {. }\end{array}$ \\
\hline $\begin{array}{l}\text { Dayal et } \\
\text { al., } 2015\end{array}$ & $\begin{array}{l}\text { Ensaio clinico } \\
\text { prospectivo } \\
\text { randomizado }\end{array}$ & 40 & $\begin{array}{l}\text { Peeling de ácido } \\
\text { salicílico a 30\% } \\
\text { versus peeling de } \\
\text { Jessner }\end{array}$ & $\begin{array}{c}6 \\
\text { (2 semanas) }\end{array}$ & $\begin{array}{l}\text { O peeling de ácido salicílico a } \\
30 \% \text { foi mais eficaz nas } \\
\text { lesões não inflamatórias ( } \mathrm{p}< \\
0,05 \text { ) e na melhora de um } \\
\text { modo geral da acne facial leve } \\
\text { a moderada. }\end{array}$ \\
\hline $\begin{array}{l}\text { Jaffary } \\
\text { et al., } \\
2016\end{array}$ & $\begin{array}{l}\text { Ensaio clínico } \\
\text { prospectivo, } \\
\text { simples-cego }\end{array}$ & 86 & $\begin{array}{l}\text { Peeling de ácido } \\
\text { pirúvico a } 50 \% \text { e do } \\
\text { ácido salicílico a } 30 \%\end{array}$ & $\begin{array}{c}8 \\
\text { (2 semanas) }\end{array}$ & $\begin{array}{l}\text { Os dois peelings foram } \\
\text { eficazes sem diferença } \\
\text { significativa }\end{array}$ \\
\hline $\begin{array}{l}\text { Lekakh, } \\
2015\end{array}$ & $\begin{array}{l}\text { Ensaio clínico } \\
\text { prospectivo, } \\
\text { randomizado }\end{array}$ & 19 & $\begin{array}{l}\text { Peeling de ácido } \\
\text { salicílico a } 30 \% \text { e } \\
\text { laser pulsado de } \\
\text { contraste }(595 \mathrm{~nm}) \\
\text { versus o peeling de } \\
\text { acido salicílico }\end{array}$ & $\begin{array}{c}3 \\
\text { (3 semanas) }\end{array}$ & $\begin{array}{l}\text { Houve uma melhora } \\
\text { significativa tanto no lado } \\
\text { com peeling de AS ( } \mathrm{p}= \\
0,001) \text { quanto no lado com } \\
\text { tratamento combinado ao } \\
\text { laser }(\mathrm{p}<0.0005) \text {. Na } \\
\text { comparação entre os } \\
\text { tratamentos, a associação do } \\
\text { laser promoveu resultados } \\
\text { melhores }(\mathrm{p}=0,003)\end{array}$ \\
\hline $\begin{array}{l}\text { Jartarkar } \\
\text { et al., } \\
2017\end{array}$ & $\begin{array}{l}\text { Ensaio clínico } \\
\text { controlado, } \\
\text { randomizado, } \\
\text { cego }\end{array}$ & 50 & $\begin{array}{l}\text { Peeling de ácido } \\
\text { salicílico a } 20 \% \text { e } \\
\text { peeling de ácido } \\
\text { mandélico a } 30 \%\end{array}$ & $\begin{array}{c}15 \\
\text { (2 semanas) }\end{array}$ & $\begin{array}{l}\text { O peeling de ácido salicílico } \\
\text { foi mais eficaz que o peeling } \\
\text { de ácido mandélico no } \\
\text { tratamento da acne } \\
\text { inflamatória, porém a } \\
\text { diferença não foi } \\
\text { estatisticamente significante } \\
\text { ( }>\text { }>0,05) \text {. No tratamento da } \\
\text { acne não inflamatória, o } \\
\text { peeling de ácido salicílico foi } \\
\text { mais eficaz que ácido } \\
\text { mandélico e a diferença foi } \\
\text { estatisticamente significativa. }\end{array}$ \\
\hline
\end{tabular}




\begin{tabular}{|l|l|l|l|c|l|}
\hline $\begin{array}{l}\text { Bae et } \\
\text { al., 2013 }\end{array}$ & $\begin{array}{l}\text { Um ensaio } \\
\text { clínico } \\
\text { randomizado } \\
\text { e simples- } \\
\text { cego }\end{array}$ & 13 & $\begin{array}{l}\text { Peeling de ácido } \\
\text { salicílico a 30\% com a } \\
\text { solução de Jessner }\end{array}$ & $\begin{array}{l}3 \\
\text { (2 semanas) })\end{array}$ & $\begin{array}{l}\text { A contagem de lesões } \\
\text { inflamatórias da acne não } \\
\text { diferiu significativamente } \\
\text { entre o ácido salicílico e a } \\
\text { solução de Jessner, embora } \\
\text { em termos de contagem de } \\
\text { lesões não-inflamatórias, o } \\
\text { lado tratado com ácido } \\
\text { salicílico mostrou melhora } \\
\text { significativa (p = 0,04), } \\
\text { enquanto que o lado com a } \\
\text { solução de Jessner não. }\end{array}$ \\
\hline
\end{tabular}

Quadro 1 - Sumarização dos estudos incluídos na revisão

Abdel e colaboradores por meio de um ensaio clínico prospectivo, unicêntrico, randomizado e duplo-cego, compararam a eficácia do peeling de ácido tricloroacético (ATA) a $25 \%$ versus peeling de ácido salicílico a $30 \%$ em 20 pacientes com acne facial leve e moderada vulgar. Todos os participantes foram submetidos ao peeling facial $25 \%$ de ATA na metade direita do rosto e $30 \%$ de ácido salicílico na metade esquerda. A cada duas semanas durante dois meses, cada paciente completou uma série de cinco sessões. O tratamento com ATA levou a uma melhora estatisticamente significativa em relação aos comedões, nas lesões inflamatórias (pápulas e pústulas) e no número total de lesões antes e depois do tratamento ( $\mathrm{p}<0,015 ; 0,000$ e 0,000$)$, respectivamente. Resultados semelhantes foram obtidos no tratamento com ácido salicílico ( $p<0,400 ; 0,000$ e 0,000). Como em relação aos comedões, a melhora foi mais frequente entre os lados da face tratada com ATA (16 pacientes) comparada com o ácido salicílico (14 pacientes) e a melhora das lesões inflamatórias foi mais frequente no lado tratado com ácido salicílico (85\%) versus $(80 \%)$ com peeling de ATA. Os autores concluíram que o ATA é superior no tratamento de comedões, enquanto o salicílico é superior no tratamento de lesões inflamatórias. Entretanto não houve diferença significativa entre os dois tratamentos, sendo os resultados semelhantes. Não foram relatadas complicações no lado tratado com peeling de ácido salicílico. Já no lado tratado com ATA, em 25\% dos indivíduos ocorreu eritema prolongado e esfoliação, e 20\% relataram hiperpigmentação (ABDEL, 2015).

Em um ensaio clínico prospectivo, randomizado, do tipo split-face, simples cego, foi avaliada a segurança e eficácia do tratamento combinado do peeling de ácido salicílico a 30\% e laser pulsado de contraste $(595 \mathrm{~nm})$ versus o peeling de acido salicílico somente. Foram incluídos 19 indivíduos com fototipo I ao III e grau de severidade da acne do III ao V, segundo 
escala de avaliação global da acne validada na Europa. Um total de três tratamentos com intervalo de três semanas foi realizado. Com base na escala de avaliação, houve uma melhora significativa tanto no lado com aplicação do peeling de AS sozinho $(\mathrm{p}=0,001)$ quanto no lado com tratamento combinado ao laser $(\mathrm{p}<0,0005)$. Na comparação entre os tratamentos, a associação do laser promoveu resultados significativamente melhores $(p=0,003)$. Não foram relatados ou observados eventos adversos menores ou mais graves em nenhum dos braços do estudo (LEKAKH et al., 2015).

Um total de cinquenta pacientes com acne de grau leve a moderadamente grave de acordo com o Índice Global de Classificação da Acne foram divididos aleatoriamente em dois grupos de 25 pacientes cada em um ensaio clínico controlado, randomizado, cego. Os pacientes do grupo A foram tratados com ácido salicílico a 20\%, e os pacientes do Grupo B foram tratados com peeling de ácido mandélico a 30\%, a cada 15 dias por seis sessões. A melhora foi classificada como leve, moderada, boa e significativa pelo investigador que foi mantido cego sobre o tratamento recebido pelos pacientes. Nos dois grupos, a melhora das lesões inflamatórias e não inflamatórias foram estatisticamente significantes $(\mathrm{p}<0,05)$. O peeling de ácido salicílico foi mais eficaz que o peeling de ácido mandélico no tratamento da acne inflamatória, porém a diferença não foi estatisticamente significante $(\mathrm{p}>0,05)$. No tratamento da acne não inflamatória, o peeling de ácido salicílico foi mais eficaz que ácido mandélico e a diferença foi estatisticamente significativa $(\mathrm{p}<0,05)$. Eritema, irritação e queimação foram os efeitos colaterais mais comuns em ambos os grupos. Em dois pacientes, ocorreram hiperpigmentação pós-inflamatória no grupo A (JARTARKAR et al., 2017).

Foi possível observar que o peeling de ácido salicílico é seguro e efetivo no tratamento da acne facial, demonstrando melhora significativa em todos os estudos incluídos. O efeito do AS nas lesões não-inflamatórias (comedões) é principalmente devido à sua atividade lipofílica e forte efeito comedolítico. Além disso, sua atividade antiinflamatória torna-o útil na redução rápida do eritema facial e uma opção também no tratamento das lesões inflamatórias.

As sessões de tratamtento nos estudos variaram de 3 a 10 semanas com intervalo de uma a duas semanas, e as complicações mais comuns relatadas foram queimação, vermelhidão e descamação. Essas reações são consideradas normais tendo em vista a natureza do procedimento. Em dois estudos (DAYAL et al., 2015; JARTARKAR et al., 2017) foi observada hiperpigmentação pós-inflamatória que ocorreu apenas em três pacientes ao todo. 
O peeling de ácido salicílico mostrou resultados semelhantes quando comparado a outros peelings como o de ATA e ácido pirúvico e a outros procedimentos como a fototerapia (ABDEL, 2015; JAFFARY et al., 2016; ALBA et al., 2016). Entretanto, em relação ao tratamento de pústulas, a fototerapia mostrou resultados melhores, possivelmente devido às suas propriedades antiinflamatórias e cicatrizantes. Mostrou resultados melhores quando comparado ao peeling com solução de Jessner (DAYAL et al., 2016; BAE et al., 2013) e ao ácido mandélico (JARTARKAR et al., 2017). Sua associação ao laser pulsado de contraste (595 $\mathrm{nm}$ ) pode promover melhores resultados (LEKHAH et al., 2015).

\section{Conclusão}

O peeling de ácido salicílico pode contribuir de maneira eficaz no tratamento da acne, tanto de lesões não inflamatórias, como os comedões, quanto inflamatórias, como pápulas e pústulas. Como a acne traz insatisfações ao indivíduo acometido, este peeling é uma opção de tratamento efetivo e seguro, como evidenciado nos estudos encontrados. Nenhum efeito adverso mais grave além de vermelhidão, eritema e descamação foram observados. Dada à natureza do procedimento, todas essas reações são consideradas normais, desde que não sejam prolongadas.

O peeling de ácido salicílico mostrou resultados semelhantes quando comparado a outros peelings como o de ATA, ácido pirúvico e a fototerapia e ainda, resultados melhores quando comparado ao peeling com solução de Jessner e ácido mandélico. Por ser de custo mais baixo e acessível, pode ainda ser uma alternativa a outros tipos de terapia como o laser ou ainda ser associado a este, para obtenção de melhores resultados.

É importante ressaltar que são necessários estudos envolvendo um número maior de indivíduos, além da inclusão de diferentes fototipos de pele para que a terapia seja incorporada com maior segurança no mercado estético. 


\section{Referências}

ABDEL M.M.A; ELAZIZ, A.D.D.A; OMAR, H. Trichloroacetic Acid Versus Salicylic Acid in the Treatment of Acne Vulgaris in Dark-Skinned Patients, v. 41, p. 1398-1404, 2015.

AL-TALIB, H. et al. Efficacy and safety of superficial chemical peeling in treatment of active acne vulgaris. An Bras Dermatol, v. 92, n. 2, p. 212-6, 2017.

ALBA, M.N. et al. Clinical comparison of salicylic acid peel and LED-Laser phototherapy to the treatment of acne vulgaris in teenagers, Journal of Cosmetic and Laser Therapy.

ARAUJO, A.P.; DELGADO, D.C.; MARÇAL, R. Acne tipologias e formas de tratamento. Revista Médica da Santa Casa de Maceió, Maceió, v. 1, n. 1, p. 26-29, 2011.

ARIF, T. Salicylic acid as a peeling agent: a comprehensive review. Clin Cosmet Investig Dermatol.v.8, p. 455-461, 2015.

ASSOCIAÇÃO BRASILEIRA DA INDÚSTRIA DE HIGIENE PESSOAL, PERFUMARIA E COSMÉTICOS - ABIHPEC. Anuário ABIHPEC 2016. Disponível em: $<$ https://abihpec.org.br/ABIHPEC_2016/Anuario2016_DIG.html\#p=55>. Acesso em $07 \mathrm{de}$ fev. 2018.

BAE, B.G., et al. Salicylic acid peels versus Jessner's solution for acne vulgaris: A comparative study. Dermatol Surg, v. 39, n.2, p. 248-253, 2013.

BAUMANN, L., SAGHARI, S. Chemical peels. In: Baumann L, Saghari S, Weisberg E, editors. Cosmetic Dermatology: Principles and Practice. 2nd ed. New York, NY, USA:

McGraw-Hill Companies; 2009.

BAGATIN, E.; HASSUN, K., TALARICO, S. Revisão sistemática sobre peelings químicos. Surgical \& Cosmetic Dermatology, v. 1, n. 1, p. 37-46 , 2009.

DAYAL, S. et al. Jessner's solution vs. $30 \%$ salicylic acid peels: a comparative study of the efficacy and safety in mild-to-moderate acne vulgaris, J Cosmet Dermatol. v.16, n. 1, p. 43$51,2017$.

FASIH, S., ARIF, A.B. Salicylic acid peeling in the treatment of Facial acne vulgaris. Pak $J$ Physiol, v.12, n.4, 2016.

GUPTA, A. et al. Quality of life in acne vulgaris: Relationship to clinical severity and demographic data. Indian J Dermatol Venereol Leprol, v. 82, n. 3, p. 292-297, 2016.

JAFFARY, F. et al. Comparison the effectiveness of pyruvic acid 50\% and salicylic acid 30\% in the treatment of acne. J Res Med Sci, v. 21, p. 31, 2016. 
JARTARKAR, S.R., et al. A randomized, single-blind, active controlled study to compare the efficacy of salicylic acid and mandelic acid chemical peel in the treatment of mild to moderately severe acne vulgaris. Clin Dermatol Rev, v.1, n. 1, p.15-18, 2017.

KATARIA, U., CHHILLAR, D. Acne: Etiopathogenesis and its management. International Archives of Integrated Medicine, v. 2, n. 5, p. 225-231, 2015.

KHUNGER, N., KUMAR, C. A clinico-epidemiological study of adult acne: Is it different from adolescent acne?. Indian J Dermatol Venereol Leprol, v. 78, n. 3, p.335-41, 2012.

KONTOCHRISTOPOULOS, G.; PLATSIDAKI, E. Chemical peels in active acne and acne scars, Clin Dermatol, v. 35, n. 2, p. 179-182, 2017.

LEKAKH, O. et al. Treatment of Acne Vulgaris With Salicylic Acid Chemical Peel and Pulsed Dye Laser: A Split Face, Rater-Blinded, Randomized Controlled Trial. J Lasers Med Sci, v.6, n. 4, p.167-170, 2015.

SELORES, M. - Acne. Nascer e Crescer, v. 20, n.3, p.SI88-SI91, 2011.

SHARQUIE, K.E., NOAIMI, A.A., AL-JANABI, E.A. Treatment of Active Acne Vulgaris by Chemical Peeling Using 88\% Lactic Acid. Our Dermatol Online, v. 5, n. 4, p. 337- 42, 2014.

TOSTI, A., GRIMES, P.E., PADOVA, M.P. Color Atlas of Chemical Peels. 2nd ed. New York, NY, USA: Springer-Verlag; 2006.

VILAR, G.N., SANTOS, L.A., SOBRAL FILHO, J.F. Quality of life, self-esteem and psychosocial factors in adolescents with acne vulgaris. An Bras Dermatol, v. 90, n. 5, p.622$29,2015$.

YOKOMIZO, V.M.F., et al. Peelings químicos: revisão e aplicação prática. Surg Cosmet Dermatol, v. 5, n.1, p. 58-68, 2013.

\section{Como citar este artigo (Formato ABNT):}

CUNHA, Bruna Luana Sousa; FERREIRA, Lilian Abreu Ferreira. Peeling de Ácido Salicílico no Tratamento da Acne: Revisão Baseada em Evidências Clínicas. Id on Line Rev.Mult. Psic., 2018, vol.12, n.42, p. 383-398. ISSN: 1981-1179.

Recebido: 14/09/2018;

Aceito: $19 / 09 / 2018$ 